\title{
The Efficacy of Ultraviolet Irradiation on Trichophyton Species Isolated From Nails
}

\author{
Ahmad Reza Nematollahi ${ }^{1}$; Parisa Badiee ${ }^{2, *}$; Ensieh Nournia ${ }^{1}$ \\ ${ }^{1}$ Department of Physical Medicine and Rehabilitation, Shiraz University of Medical Sciences, Shiraz, IR Iran \\ ${ }_{2}^{2}$ Professor Alborzi Clinical Microbiology Research Center, Shiraz University of Medical Sciences, Shiraz, IR Iran \\ *Corresponding author: Parisa Badiee, Professor Alborzi Clinical Microbiology Research Center, Shiraz University of Medical Sciences, Shiraz, IR Iran. Tel: +98-7136474292, \\ Fax:+98-7136474303, E-mail: badieep@sums.ac.ir
}

Received: February 11, 2014; Revised: June 7, 2014; Accepted: June 8, 2014

\begin{abstract}
Background:The treatment of onychomycosis is a challenge and infections are typically more severe and difficult to treat in toenails than in fingernails.

Objectives: The current study aimed to investigate the fungicidal effect of ultraviolet radiation on the growth of dermatophytes isolated from nails.

Patients and Methods: Samples from patients with clinical manifestations of onychomycosis were inoculated onto Sabouraud dextrose agar and incubated at $30^{\circ} \mathrm{C}$ for 14 days. Isolated species were identified by specific laboratory examinations; UV-A, UV-B, and UV-C light were used to irradiate two strains of Trichophyton mentagrophytes and T. rubrum. Colony count, size and growth rate of the isolated fungi were evaluated under laboratory conditions.

Results: Trichophyton rubrum type 1 was less sensitive to UV-A and UV-C, and more sensitive to UV-B than type 2. T. mentagrophytes type 2 was slightly responsive to UV-A therapy, although no decrease in colony count was observed. Increased doses of UV-B and UV-C irradiation decreased the counts. The effect of radiation on colony size was dependent on the dose and type of irradiation.

Conclusions: UV-A, UV-B, and UV-C light seem to be effective in decreasing colony growth of the most prevalent fungi, which caused onychomycosis in the current study samples. Further studies are needed to determine the efficacy of ultraviolet light therapy, identify possible side effects, and establish appropriate dosages for the antifungal effect of this therapy.
\end{abstract}

Keywords: Dermatophyte; Trichophyton; Onychomycosis; Ultra-violet Light

\section{Background}

Dermatophytosis, one of the most common infectious diseases in the world, can be caused by dermatophyte fungal species of the genera Trichophyton, Microsporum and Epidermophyton. Onychomycosis is a fungal infection of the nails, which may be caused by many types of fungi, other dermatophytes or yeast species (1). Treatment of onychomycosis is a challenge and infections are typically more severe and difficult to treat in toenails than in fingernails (1). Optimal treatment of onychomycosis is extensively discussed. Treatment requires long-term therapy with oral antifungal medication (with the cure rates ranging from $48 \%$ to $76 \%$ ) which may have side effects, and/or topical agents such as ciclopirox (34\% cure rate) $(2,3)$. Oral therapies (terbinafine, itraconazole) provide access to the nail bed and matrix and are also used to treat concomitant skin infections such as tinea pedis. To prevent side effects prior to and periodically during oral therapy, liver function should be controlled. The newer imidazoles may lead to a higher cure rate (4), but these drugs are considerably more expensive than routine treatments. Topical thera- pies are associated with low rates of adverse events and mild localized reactions at the application site, but due to inadequate penetration, especially when the nails are thick, their efficacy is limited.

UV radiation induces DNA damage leading to inactivation of the pathogenic fungus commonly measured by viability count. UV-A with long wavelength (315 - $400 \mathrm{~nm}$ ) rays has a slow effect on the skin and is poorly absorbed by the ozone layer. This ray is 1,000 times more common than UV-B with the middle-range of 290 - $320 \mathrm{~nm}$ that causes visible damage, commonly observed as redness and blistering and are a major cause of sunburn and skin cancer (5). UV-C with wavelengths less than $290 \mathrm{~nm}$ (100 - $280 \mathrm{~nm}$ ) is the shortest and has the highest UV energy. It is filtered by the ozone layer; these wavelengths do not reach the earth surface and do not penetrate the atmosphere. Its rays are the most powerful, and do not damage human skin. UV-A radiation can penetrate glass and clouds. Thus, people are exposed to large doses of UV-A throughout their life, but UV-B rays do not penetrate glass (5). High doses of UV-A and UV-B radiation have

Copyright (C) 2015, Ahvaz Jundishapur University of Medical Sciences. This is an open-access article distributed under the terms of the Creative Commons Attribution-NonCommercial 4.0 International License (http://creativecommons.org/licenses/by-nc/4.0/) which permits copy and redistribute the material just in noncommercial usages, provided the original work is properly cited. 
significant inhibitory effects on dermatophytes (6). The antibacterial activity of UV-C radiation reduces the number of bacteria on environmental surfaces and vegetative bacteria including Mycobacterium tuberculosis, viruses and fungi $(7,8)$.

\section{Objectives}

The current study aimed to investigate the fungicidal effect of UV radiation on the growth of dermatophytes isolated from nails, considering the low efficacy of current treatment options and ease of exposing nails to UV light.

\section{Patients and Methods}

The sample comprised all the patients referred to the dermatology clinic (Shahid Faghihi hospital, Shiraz University of Medical Sciences, Iran) with suspected onychomycosis. The inclusion criteria were clinical manifestations such as distal or proximal sub ungula onychomycosis, white superficial onychomycosis, pitting, paronychia, onycholysis and nail plate discoloration. All diagnoses were confirmed by the mycology laboratory of Shahid Faghihi Hospital, Shiraz University of Medical Sciences, Iran. Since this procedure was part of the patients' usual treatment protocol, informed consent for research purposes was not specifically sought. Fragments of nail plate and nail bed scrapes were inoculated onto Sabouraud dextrose agar (SDA) (Merck, Germany) with the antimicrobials cycloheximide (Sigma-Aldrich, Germany) and chloramphenicol (Merck, Germany) (9), and incubated at $30^{\circ} \mathrm{C}$ for 14 days. Isolated species were identified by macroscopic examination of colony morphology (size, color of the colonies from the top and bottom of the plate, and growth rate). Microscopic examinations were performed to identify dermatophyte species using lacto phenol-cotton blue and diagnostic methods such as growth on trichophyton agar media (Quelab, UK) and rice, and hair perforation test were performed to identify (9).

To determine the influence of different doses of UV light on the fungicidal effect, colony count and size, and growth rate of the isolated fungi were evaluated under laboratory conditions. Two strains of Trichophyton mentagrophytes and two strains of T. rubrum were isolated from the patients and used in the current study. Trichophyton rubrum type 1 was white and had fluffy colony with pink to burgundy on reverse. Type 2 was granular with powdery-velvet, radially folded and pale brown in color and the reverse was dark tan. T. mentagrophyte type 1 was downy, floccose and white in color with the fade yellow on reverse. Finally, type 2 was coarsely granular and light buff in color with the buff-tan color on reverse.

As in the skin samples from patients, only mycelium can be observed, in the current study mycelial colonies were harvested, suspended in Sabouraud dextrose broth (SDB) (Merck, Germany) and dispersed to small part suspensions using a sonic dismembrator (Dr. Hielscher GmbH,
Stahnsdorf, Germany). Cell densities of the suspensions were counted in a Neubauer hemocytometer (Hausser Scientific, Horsham, PA, USA), and reached $10^{5}$ pieces/ $\mathrm{mL}$. Fifty $\mu \mathrm{L}$ of suspension was inoculated onto SDA to observe macroscopic and microscopic colony characteristics. In each test, a $10 \mathrm{~mL}$ aliquot of the dermatophyte suspension was inoculated onto a petri dish. UV-A and UV-B lights were supplied by a Waldmann UV801KL apparatus (Waldmann, Villingen-Schwenningen, Germany) at $470 \mathrm{~W}$, and UV-C light was supplied by an F30T8/ GL apparatus (Young deungpo-Gu, Seoul, South Korea) at 30 W. Five different doses of UV light were used: UV-A (315 - 400) 3, 6, 9, 12, $15 \mathrm{~J} / \mathrm{cm}^{2}$, UV-B (280 - 315) 0.5, 1, 1.5, 2, $2.5 \mathrm{~J} / \mathrm{cm}^{2}$ and UV-C (100 - 280) 0.3, 0.6, 0.9, 1.2, $1.5 \mathrm{~J} / \mathrm{cm}^{2}$. One positive control without exposure to UV light was cultured in each round with the test samples. The distance between the UV light source and the sample was $30 \mathrm{~cm}$. This distance is widely used in dental and cosmetic fields to sterilize tools (10). In the current study, for UV-A and UV-B radiation, the lamps were utilized in the centers for dermatological diseases in order to obtain some practical implications.

Since radiation reduces the volume and raises the count because of evaporation, which may lead to errors, the specimen volumes were made up to the primary volume $(10 \mathrm{~mL})$ after irradiation, with SDB. For each irradiated specimen, the fungicidal effect of UV was evaluated by preparing five cultures to incubate at $30^{\circ} \mathrm{C}$ under laboratory conditions; therefore, 75 cultures for each fungus were prepared. Colony size, morphology and count, and growth rate of all isolates were checked in the plates every two days and mean values were calculated. Since most colony growth occurs during the first 14 days, the results were graphed after this period. All statistical analyses were conducted using Microsoft Excel software.The current research conformed to the Helsinki Declaration and local legislation, and was approved by the local ethics committee (91-5) in Clinical Microbiology Research Center, Shiraz University of Medical Sciences.

\section{Results}

In response to the UV treatments, compared with suspension control without irradiation, colony morphology for each dermatophyte remained unchanged, but colony count and size changed depending on the species and type of isolate. Of the two T. rubrum isolates (Figure 1), type 1 was less sensitive to UV-A and UV-C, and more sensitive to UV-B. Continuous irradiation with the UV-A and UV-B doses used in the study did not completely suppress the growth of the isolates, but higher intensities of UV-C light inhibited the growth of T. rubrum.

Of the two T. mentagrophytes isolates (Figure 2), colony count in type 1 decreased as the dose of all three types of UV radiation increased. However, colony count in T. mentagrophytes type 2 showed different patterns as the dose of UV radiation increased. Type 2 strain was slightly responsive to UV-Abut compared to control cultures; there 
was no significant effect on colony count. Neither UV-B nor UV-C decreased the type 2 colony counts at low doses, but higher doses led to decreases in colony counts.

According to Table 1, UV-A irradiation led to a slight increase in colony size in the four strains except T. mentagrophytes type 1 , in which colony size increased with the lowest dose of irradiation and significantly decreased with increasing the doses. Irradiation with UV-B increased colony size in T. mentagrophytes type 2 , but in type $1 \mathrm{UV}$ ray increased colony size at the lowest dose and decreased colony size with increasing doses. UV-B irradiation decreased colony size in T. rubrum type 2 but had no significant effect on type 1 or either of the two strains of T. mentagrophytes. Irradiation with UV-C decreased colony size except for T. mentagrophytes type 2, in which higher doses increased colony size.

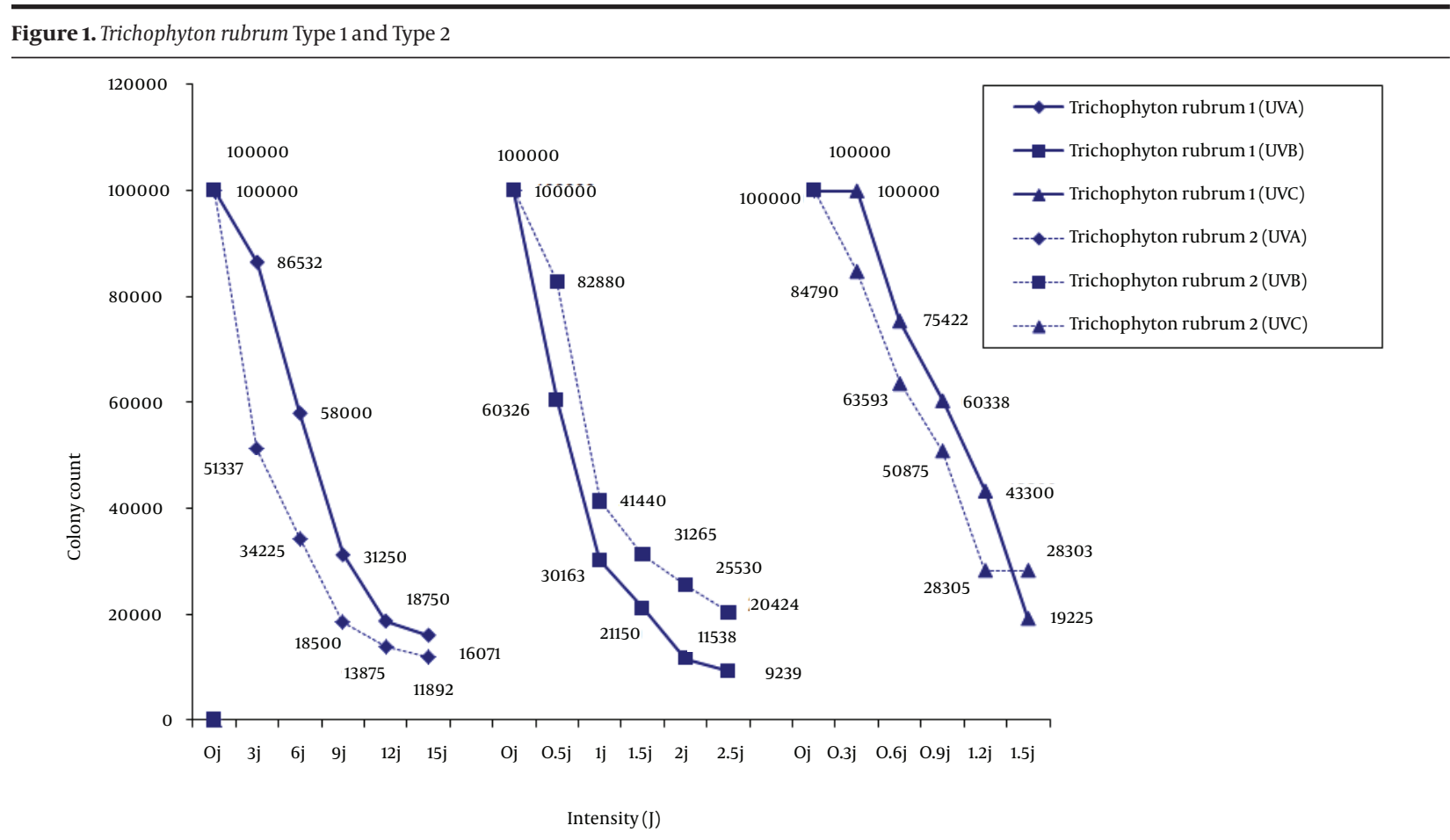

Mean colony counts at different intensities UV-A (315-400 nm), UV-B (290-320 nm) and UV-C (100-280nm).

Figure 2. Trichophyton mentagrophytes Type 1 and Type 2

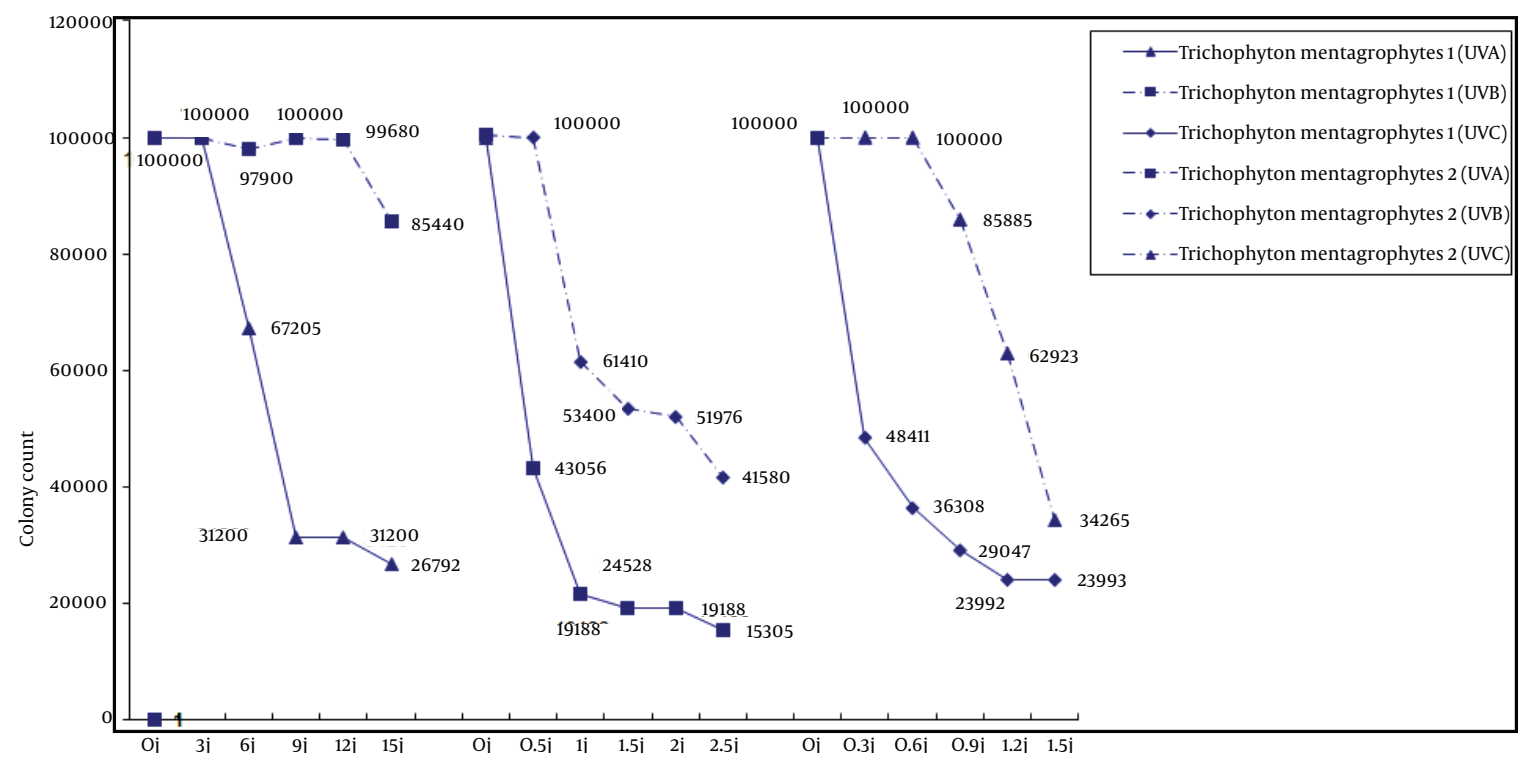

Mean colony counts at different intensities of UV-A (315-400 nm), UV-B (290-320 nm) and UV-C (100-280 nm). 
Nematollahi AR et al.

\begin{tabular}{|c|c|c|c|c|}
\hline UV light ${ }^{b}$ & Trichophyton mentagrophyte $\mathbf{1}$ & T. mentagrophyte $\mathbf{2}$ & T. rubrum 1 & T. rubrum $\mathbf{2}$ \\
\hline UVA 0 & $14 \pm 1.1$ & $13 \pm 1.1$ & $12 \pm 1.1$ & $23 \pm 2$ \\
\hline UVA 3 & $20 \pm 1.5$ & $13 \pm 1.1$ & $13 \pm 1.1$ & $23 \pm 1.9$ \\
\hline UVA 6 & $20 \pm 1.6$ & $13 \pm 1.1$ & $13 \pm 1.1$ & $23 \pm 2$ \\
\hline UVA 9 & $7 \pm 0.8$ & $14 \pm 1.1$ & $13 \pm 1.1$ & $26 \pm 2$ \\
\hline UVA 12 & $4 \pm 0.5$ & $15 \pm 1.2$ & $13 \pm 1.1$ & $26 \pm 1.9$ \\
\hline UVA 15 & $3 \pm 0.5$ & $15 \pm 1.2$ & $13 \pm 1.1$ & $26 \pm 1.9$ \\
\hline UVB 0 & $14 \pm 1.1$ & $13 \pm 1.1$ & $12 \pm 1.1$ & $23 \pm 1.8$ \\
\hline UVB 0.5 & $22 \pm 1.6$ & $16 \pm 1.2$ & $13 \pm 1.1$ & $17 \pm 1.3$ \\
\hline UVB 1 & $20 \pm 1.6$ & $16 \pm 1.2$ & $13 \pm 1.1$ & $16 \pm 1.3$ \\
\hline UVB 1.5 & $9 \pm 1$ & $20 \pm 1.9$ & $12 \pm 1.1$ & $10 \pm 1.0$ \\
\hline UVB 2 & $6 \pm 0.5$ & $20 \pm 1.9$ & $12 \pm 1.1$ & $10 \pm 1.0$ \\
\hline UVB 2.5 & $5 \pm 0.5$ & $20 \pm 2$ & $11 \pm 1.1$ & $8 \pm 0.9$ \\
\hline UVC 0 & $14 \pm 1.1$ & $13 \pm 1.1$ & $12 \pm 1.1$ & $23 \pm 2$ \\
\hline UVC 0.3 & $12 \pm 1.1$ & $9 \pm 0.9$ & $8 \pm 0.9$ & $20 \pm 1.9$ \\
\hline UVC 0.6 & $11 \pm 1.0$ & $9 \pm 0.9$ & $8 \pm 0.9$ & $20 \pm 1.9$ \\
\hline UVC 0.9 & $10 \pm 1.0$ & $8 \pm 0.9$ & $8 \pm 0.9$ & $20 \pm 1.9$ \\
\hline UVC 1.2 & $7 \pm 0.9$ & $20 \pm 1.9$ & $8 \pm 0.9$ & $20 \pm 1.9$ \\
\hline UVC 1.5 & $6 \pm 0.8$ & $20 \pm 1.9$ & $7 \pm 0.9$ & $20 \pm 2$ \\
\hline
\end{tabular}

${ }^{a}$ Values are presented as mean \pm SD

$\mathrm{b}$ Values are presented as $\mathrm{J} / \mathrm{cm}^{2}$.

\section{Discussion}

According to obtained data, UV light can affect colony count and size of T. rubrum and T. mentagrophytes in the culture, the two fungal species frequently isolated from patients with onychomycosis. The estimated prevalence of onychomycosis caused by these species ranges from 10 to $21.3 \%$ in the general population in Australia, the United Kingdom and Iran (11, 12). This prevalence rate may increase to $30 \%$ in specific populations such as people over 70 years old (13-15). It is challenging to determine the optimal treatment and circumventing drug resistance in pathogenic fungi, including dermatophytes. Since the nails and skin are being exposed to sunlight, many photodynamic treatments are developed in dermatologic studies. For example, UV light therapy is investigated in diseases such as scleroderma (16) and onychomycosis (17).

The mechanism of UV radiation is the primary photochemical reaction, which damages DNA and affects survival and rate of germination (18). This molecular change makes the DNA unstable for essential biological processes such as transcription and replication. As a rule, the simpler the microorganism is in anatomical terms, the more easily it is inactivated by UV radiation. This is why viruses and prokaryotic cells such as bacteria are more easily destroyed than a complex of microorganisms such as eukaryotic cells, yeasts and vegetative fungi. In particular, fungal spores in which the DNA is protected by a concentrated cytoplasm and pigmented cell wall need high doses of UV energy to be destroyed.

Some data suggest that solar UV radiation can inhibit the growth of fungi on the soil surface in the antarctic terrestrial environment (19), and has disinfectant effects that can vary depending on the type and dosage of irradiation, condition of the surface and exposure time. The effective exposure time was reported to be 45 minutes for yeast and 75 minutes for mold (20). Different doses of UV-B light show different effects on the growth of Candida albicans. Increasing doses of UV-B strongly reduced hyphal growth in this fungus and triggered enhanced blastospore formation (21). The mechanism of resistance to UV radiation is not completely known, but intra hyphal growth and recolonization of old cells by new ones were observed in all the strains investigated by Gorbushina et al. (22).

In the present study, UV-C radiation affected T. rubrum type 1: as the dose increased, colony count decreased. However, supplementary therapy may still be needed $(7,8)$. Irradiation with UV-A and UV-B also decreased the colony count of some strains tested in the current study which was proportional to the dose. This finding may be due to the synthesis of new hyphae through the old hyphae not exposed to irradiation, or may be related to the age of the exposed hyphae (i.e. recolonization of older hyphae) in the suspension. Trichophyton rubrum is one of the most frequent causal agents in nail infections, and recur- 
rence of the infection and resistance to therapy are the most important limitations to treatment success for this anthropophilic dermatophyte (22). This species was unaffected by infrared irradiation, responded with increased pigmentation to UV-A at13.5 $/ \mathrm{cm}^{2}$ twice daily, and was inhibited by a single dose of UV-B at $15.1 \mathrm{~J} / \mathrm{cm}^{2}(6)$.

Trichophyton mentagrophytes may be anthropophilic or zoophilic in nature. The anthropophilic type causes chronic infection but the zoophilic type causes inflammatory lesions. Growth rates, surface colony color and the texture of colony morphology are different in each strain. The current study compared two strains of T. mentagrophytes. According to Figure 2, UV-A, UV-B and UV-C had similar effects on T. mentagrophytes type 1, but affecting type 2 strains differently. The negative phototropism of dermatophytes were reported previously (6). Increasing pigmentation in response to UV-A $\left(13.5 \mathrm{~J} / \mathrm{cm}^{2}\right.$ twice daily) was observed in T. rubrum (6). Electron microscopy studies showed a marked thickening and blurred contours of the cell walls grown with a brightener effect related to interference by optical brighteners with the formation of normal chitin fibrils and thickening of the cell walls (23).

In the present study, increasing doses of UV-A, UV-B and UV-C irradiation increased colony size in T. mentagrophytes type 2 . It is well known that UV radiation damages human tissues, particularly the skin. UV-B irradiation of the skin is particularly well studied, and is accepted as the main cause of skin cancer. The depth of penetration of UV-C radiation into the human skin is very low; therefore, the risk of skin cancer associated with this type of treatment is low. There are studies which report the treatment of localized infections with multidrug-resistant microorganisms (24) and subungual onychomycosis (25) with UV-C light irradiation and favorable responses. As mentioned in these studies, the cases were limited and side effects should be taken into account more carefully. When UV irradiation is used to treat onychomycosis, it is important to minimize exposure by carefully masking the area with adhesive material. In addition, the dose of radiation must be minimized by careful dosimetery calculated with reference to the individual patient's nails, the etiologic agent, and optical properties.

A potential limitation of this study was the limited availability of human nail samples. If there were enough samples, by using different types and doses of irradiation such as UV-A UV-B and UV-C for every single nail sample separately, the current study could directly examine the effects of radiation on them, but since the volume of samples was low, they were just cultured after the isolation of fungal strains and it was possible to evaluate different types and doses of irradiation in the isolated strains. UVA, UV-B and UV-C seem to be effective in decreasing colony growth in some prevalent fungi, which caused onychomycosis in the patients. Further studies are needed to determine the efficacy of this therapy, identify possible side effects and establish appropriate dosages for the antifungal action of UV radiation therapy.

\section{Acknowledgements}

Authors wish to thank Dr. Hassan Khajehei, for his careful editing of the manuscript, and to thank K. Shashok (Author AID in the Eastern Mediterranean) for improving the manuscript.

\section{Authors' Contributions}

Study design and data analysis: Parisa Badiee ; sampling and data acquisition: Ensieh Nournia; tests interpretation and manuscript drafting: Ahmad Reza Nematollahi. All authors read and approved the final manuscript.

\section{Funding/Support}

The present article is based on research done in partial fulfillment of the requirements for the thesis by Ensieh Nounia, and was financially supported by Shiraz University of Medical Sciences through grant No. 89-01-0102328.

\section{References}

1. Gupta AK, Cooper EA. A Simple Algorithm for the Treatment of Dermatophyte Toenail Onychomycosis. Skin Ther Lett com. 2008;4:1-3.

2. Gupta AK, Ryder JE, Johnson AM. Cumulative meta-analysis of systemic antifungal agents for the treatment of onychomycosis. Br J Dermatol. 2004;150(3):537-44.

3. Gupta AK, Joseph WS. Ciclopirox $8 \%$ nail lacquer in the treatment of onychomycosis of the toenails in the United States. J Am Podiatr Med Assoc. 2000;90(10):495-501.

4. Hainer BL. Dermatophyte infections. Am Fam Physician. 2003;67(1):101-8.

5. Boniol M, Autier P, Boyle P, Gandini S. Cutaneous melanoma attributable to sunbed use: systematic review and meta-analysis. BMJ. 2012;345:e4757.

6. Brasch J, Menz A. UV susceptibility and negative phototropism of dermatophytes. Mycoses. 1995;38(5-6):197-203.

7. Smijs TG, van der Haas RN, Lugtenburg J, Liu Y, de Jong RL, Schuitmaker HJ. Photodynamic treatment of the dermatophyte Trichophyton rubrum and its microconidia with porphyrin photosensitizers. Photochem Photobiol. 2004;80(2):197-202.

8. Chang JC, Ossoff SF, Lobe DC, Dorfman MH, Dumais CM, Qualls RG, et al. UV inactivation of pathogenic and indicator microorganisms. Appl Environ Microbiol. 1985;49(6):1361-5.

9. Habif TP. Clinical dermatology.Edinburg: Mosby, Elsevier; 2010.

10. Santos C, Paterson RR, Venancio A, Lima N. Filamentous fungal characterizations by matrix-assisted laser desorption/ ionization time-of-flight mass spectrometry. J Appl Microbiol. 2010;108(2):375-85.

11. Ghannoum MA, Hajjeh RA, Scher R, Konnikov N, Gupta AK, Summerbell R, et al. A large-scale North American study of fungal isolates from nails: the frequency of onychomycosis, fungal distribution, and antifungal susceptibility patterns. J Am Acad Dermatol. 2000;43(4):641-8.

12. Rezaei-Matehkolaei A, Makimura K, de Hoog S, Shidfar MR, Zaini F, Eshraghian M, et al. Molecular epidemiology of dermatophytosis in Tehran, Iran, a clinical and microbial survey. Med Mycol. 2013;51(2):203-7.

13. Erbagci Z, Tuncel A, Zer Y, Balci I. A prospective epidemiologic survey on the prevalence of onychomycosis and dermatophytosis in male boarding school residents. Mycopathologia. 2005;159(3):347-52. 
14. de Berker D. Clinical practice. Fungal nail disease. $N$ Engl J Med. 2009;360(20):2108-16.

15. Gupta AK, Jain HC, Lynde CW, Macdonald P, Cooper EA, Summerbell RC. Prevalence and epidemiology of onychomycosis in patients visiting physicians' offices: a multicenter canadian survey of 15,000 patients. JAm Acad Dermatol. 2000;43(2 Pt 1):244-8.

16. Kerscher M, Volkenandt M, Gruss C, Reuther T, von Kobyletzki G, Freitag M, et al. Low-dose UVA phototherapy for treatment of localized scleroderma. J Am Acad Dermatol. 1998;38(1):21-6.

17. Landsman AS, Robbins AH, Angelini PF, Wu CC, Cook J, Oster M et al. Treatment of mild, moderate, and severe onychomycosis using 870- and 930-nm light exposure. J Am Podiatr Med Assoc. 2010;100(3):166-77.

18. Ravanat JL, Douki T, Cadet J. Direct and indirect effects of UV radiation on DNA and its components. J Photochem Photobiol B. 2001;63(1-3):88-102.

19. Hughes KA, Lawley B, Newsham KK. Solar UV-B radiation inhibits the growth of Antarctic terrestrial fungi. Appl Environ Microbiol. 2003;69(3):1488-91.
20. Ozcelik B. Fungi/Bactericidal and Static Effects of Ultraviolet Light in 254 and $354 \mathrm{~nm}$ Wavelengths. Res J Microbiol. 2007;2(1):42-9.

21. Brasch J, Kay C. Effects of repeated low-dose UVB irradiation on the hyphal growth of Candida albicans. Mycoses. 2006;49(1):1-5.

22. Gorbushina AA, Whitehead K, Dornieden T, Niesse A, Schulte A Hedges JI. Black fungal colonies as units of survival: hyphal mycosporines synthesized by rock-dwelling microcolonial fungi. Can J Bot. 2003;81(2):131-8.

23. Brasch J, Kreiselmaier I, Christophers E. Inhibition of dermatophytes by optical brighteners. Mycoses. 2003;46(3-4):120-5.

24. Dai T, Vrahas MS, Murray CK, Hamblin MR. Ultraviolet C irradiation: an alternative antimicrobial approach to localized infections? Expert Rev Anti Infect Ther. 2012;10(2):185-95.

25. Boker A, Rolz-Cruz G, Cumbie B, Kimball AB. A single-center prospective, open-label, pilot study of the safety, local tolerability, and efficacy of ultraviolet-C (UVC) phototherapy for the treatment of great toenail onychomycosis. J Am Acad Dermatol. 2007;58(2):AB82. 